\title{
Sperm cryopreservation in cancer patients: a review Sperm cryopreservation and cancer
}

\author{
Criopreservação de espermatozóides em pacientes com câncer: \\ revisão da literatura Criopreservação de espermatozóides e câncer
}

\author{
Daniela Paes de Almeida Ferreira Braga, DVM, M.Sc. ${ }^{1,2}$,Amanda Souza Setti, B.Sc. ${ }^{1,2}$,Assumpto Iaconelli Jr., MD ${ }^{1,2}$,Edson Borges \\ Jr., MD, Ph.D ${ }^{1,2}$ \\ ${ }^{1}$ Fertility - Centro de Fertilização Assistida \\ ${ }^{2}$ Associação Instituto Sapientiae - Centro de Estudos e Pesquisa em reprodução humana assistida
}

\begin{abstract}
RESUMO
Devido aos avanços notáveis no tratamento do câncer infantil, vimos grandes melhorias na esperança de vida, com uma sobrevida de até $80 \%$ das crianças, o que resulta em um aumento, em longo prazo, da população de adultos sobreviventes às doenças malignas infantis. Embora os tratamentos oncológicos sejam altamente eficazes, uma grande preocupação é seu impacto adverso sobre a fertilidade. Nesta revisão, vamos resumir a literatura sobre a criopreservação de espermatozóides em pacientes com câncer.
\end{abstract}

Palavras-chave: câncer, espermatozóide, criopreservação, fertilidade.

\section{ABSTRACT}

Due to remarkable advances in the treatment of childhood cancer, we have seen great improvements in life expectancy, with up to $80 \%$ of children surviving their disease, resulting in a growing population of adult long-term survivors of childhood malignancies. Although oncological treatments are highly effective, a major concern is their adverse impact on fertility. In this review we will summarize the literature regarding sperm cryopreservation in cancer patients.

Keywords: cancer, sperm, cryopreservation, fertility.

\section{INTRODUCTION}

Cancer is the leading cause of death in the world, accounting for 7.6 million mortality cases in 2005. Cancer treatments (surgery, radiotherapy and chemotherapy) are undertaken to remove malignancies, prolong the patient's life and improve their quality of life.

Due to remarkable advances in the treatment of childhood cancer, we have seen great improvements in life expectancy, with up to $80 \%$ of children surviving their disease, resulting in a growing population of adult long-term survivors of childhood malignancies (Brenner et al. 2007). It is therefore estimated that, by the end of 2012, 1 in 250 young adults aged 20-29 years will be a cancer survivor. Although oncological treatments are highly effective, a major concern is their adverse impact on fertility. Currently, available drugs to prevent testicular damage from cytotoxic therapy have not proved helpful in humans so far. However, improved therapeutic regimens using less gonadotoxic protocols could enable spontaneous recovery of spermatogenesis, but their use is not always possible without compromising patient survival.

In fact, since rapidly dividing cells are the target of chemoand radiotherapy, these treatments act not only on cancer cells, but also on germ cells. Damage to reproductive func- tion is a very frequent and well documented side effect associated with the treatment of malignant tumors. The first work describing chemotherapy-induced azoospermia was published in 1948 (Spitz 1948) and it has been suggested that the effects might be transient or permanent depending upon the individual variability in the sensitivity to reproductive damage. The severity of damage is dependent on the type of chemotherapy or radiotherapy, the treatment protocol and the age and gender of the patients.

The increasing success of cancer treatment and determined efforts to improve the quality of life after successful treatment has turned attention to the preservation of reproductive function, especially in young patients.

Concern for future fertility is high among individuals newly diagnosed as having cancer. Approximately three-quarters of men and women younger than 35 years who are childless at the time of cancer diagnosis desire children in the future (Schover et al. 1999).

Fertility preservation is an emerging field that encompasses a variety of fertility therapies for patients anticipating medical treatment that could affect future reproductive outcomes. Although most frequently associated with cancer treatment, fertility preservation has also been used for medical conditions like lupus, glomerulonephritis, and myelodysplasia, as well as in adolescent females with conditions known to be associated with premature ovarian failure, such as Turner mosaicism.

Current available strategies to preserve fertility are limited and vary by age and sex. Prepuberal males options include: (i) minimizing the testicular damage from cancer treatment or protecting spermatogonial stem cells in vivo; and (ii) cryopreserving testicular tissue prior to gonadotoxic treatment in the form of either cell suspension, tissue fragments or whole organ. For postpubertal males, sperm and embryo cryopreservation are well-established and effective methods of fertility preservation.

In this review we will summarize the literature regarding sperm cryopreservation in cancer patients.

\section{FERTILITY PRESERVATION}

As discussed above, cancer therapy can result in subfertility or sterility due to gonad removal or permanent damage to germ cells from adjuvant therapy. Loss of fertility in adult life is a major psychologically traumatic consequence of cancer treatment. Indeed, in a quality-of-life analysis of former oncological patients, about $80 \%$ viewed themselves as potential parents, and the vast majority of younger cancer survivors saw their cancer experience as pivotal in preparing them to be better parents (Schover et al. 1999). Therefore, since post-therapy recovery of spermatogenesis remains unpredictable, it is important to inform patients facing infer- 
tility as a side effect of their treatment of all the options available to preserve their fertility.

In order to reduce the deleterious effects of gonadotoxic therapies, different strategies have been tested, such as testicular shielding and the use of cytoprotective drugs. Limiting radiation exposure by shielding or removing the testes from the radiation field should be implemented whenever possible. Gonadal protection through hormonal suppression is based on the principle that disruption of gametogenesis renders the gonads less sensitive to the effects of cytotoxic drugs or irradiation. Promising results were obtained in rodents (Shetty and Meistrich 2005), but not in non-human primates (Boekelheide et al. 2005) or humans (Brennemann et al. 1994; Blake et al. 2007), except in one clinical trial (Masala et al. 1997) where only moderate stem cell death was induced by chemotherapy. Anti-apoptotic agents (Otala et al. 2004; Carmely et al. 2009) and other cytoprotective substances (Lirdi et al. 2008; Okada et al. 2009) have also been used with partial success in rodents but not in humans. In conclusion, no effective gonadoprotective drugs are so far available for use in humans and studies aiming to identify factors regulating spermatogonial proliferation are therefore required to find novel targets for in vivo spermatogonial stem cells protection.

Another potential alternative for preserving fertility in prepubertal boys involves storage of testicular tissue, in the hope that future technologies will allow its safe utilization. As prepubertal testicular tissue contains spermatogonial stem cells from which haploid spermatozoa are ultimately derived, these cells can either be cryopreserved as a cell suspension or in the form of tissue.

However, although cryopreservation of testicular tissue is offered in some centers, it is still considered experimental; potential future uses include in vitro maturation of spermatogonia into spermatocytes or germ-cell transplant into native testicular tissue. Patients and their parents must be counseled that this technology is still being developed, and potential use of specimens is unlikely for several more years.

Cell suspensions have been developed with a view to facilitating cryopreservation, as cell heterogeneity in tissue pieces renders tissue freezing more challenging. Preparation of cell suspensions requires mechanical and/or enzymatic digestion of tissue, compromising cell survival and cell-to-cell interactions necessary for cell proliferation and differentiation. For human testicular cell suspensions, post-thaw viability of up to $60 \%$ was achieved (Brook et al. 2001; Hovatta 2001). However, whether it is better to produce cell suspensions before or after cryopreservation is still unclear.

Cryopreservation of testicular tissue pieces may be considered as an alternative method capable of maintaining cell-to-cell contacts between Sertoli and germinal stem cells, and therefore preserving the stem cell niche necessary for their survival and subsequent maturation. Other advantages of this method may be preservation of the Sertoli cells, since there is evidence of their reversion to a dedifferentiated state as a consequence of chemotherapy, and Leydig cells, whose preservation may be useful to alleviate the hormonal imbalance caused by cytotoxic therapy.

Because of the complexity of the tissue architecture, cryopreservation protocols must strike a balance between optimal conditions for each cellular type, depending on the water content, size and shape of cells, and the water permeability coefficient of their cytoplasmic membrane. In addition, problems can arise when extracellular ice forms, as it can cleave tissues into fragments.

None of the above mentioned approaches have proved efficient and safe in humans. The only established method to secure fertility in male cancer patients before gonadotoxic therapy is semen cryopreservation, which in time can be used for assisted reproduction technologies (ART).

\section{SPERM CRYOPRESERVATION}

Sperm cryopreservation provides a useful and effective method in infertility management for many men. In fact, cancer patients can use cryopreservation to preserve their fertility prior to treatments such as radiation and chemotherapy. The processes of freezing human semen and achie- ving successful fertilization via intrauterine insemination were established many decades ago (Bunge and Sherman 1953). At that time a successful, practical technique for cryopreservation of human spermatozoa was introduced by the demonstration that the sperm, after being frozen and stored in dry ice $(-780 \mathrm{C})$, were capable of fertilization and the subsequent induced development of normal progeny. The introduction in 1963 of a method for freezing human semen in liquid nitrogen vapor and its storage at $-1960 \mathrm{C}$ was followed by reports of normal births with its use. The basic principles of technique refined since 1953 have proved suitable for the establishment of clinical cryobanks, which have resulted in normal, healthy offspring in various parts of the world.

During the last 30 years several improvements in sperm cryobanking have occurred. Storage in liquid nitrogen has become the standard and extenders containing cryoprotectants have been added to the medium. These extenders have several functions, including: (i) optimizing osmotic pressure and $\mathrm{pH}$, (ii) providing an energy source to prevent undesirable use of intracellular sperm phospholipid, (iii) preventing bacterial contamination by including an antibiotic and (iv) allowing for semen dilution while offsetting the deleterious effect on survival produced by high dilution.

Before the in vitro fertilization (IVF)/intracytoplasmic sperm injection (ICSI) era, cryopreserved semen was used only for intrauterine inseminations. However, because of the deleterious effects of the freezing and thawing of the semen, the post-thaw quality was often not good enough for intrauterine inseminations. The introduction of new, sophisticated ART during the last decades such as IVF and ICSI overcame these severe sperm concentration and motility problems. These techniques provided patients, who banked semen and became infertile after treatment, with a reasonable chance of conception.

Although complex techniques have improved fertilization potential of the thawed sperm, the freezing and thawing methods expose spermatozoa to much physical and chemical damage, and several improvements have been made to the process of cryopreservation and thawing.

\section{SPERM CRYOPRESERVATION TECHNIQUE}

The most commonly reported detrimental effect of cryopreservation on human spermatozoa is a marked decrease in motility. It occurs despite many advances in cryopreservation methodology. The primary cause of cellular damage during cryopreservation is the formation of intracellular ice. However, cell survival depends on the nature of the suspending medium, and understanding the profound protectant effect of this medium has led to the development of numerous protective agents.

Cryoprotectants are low-molecular-weight and highly permeable chemicals that serve to protect spermatozoa from freeze damage. There are four main known cryoprotectants: glycerol, ethylene glycol, dimethyl sulphoxide and 1,2-propanediol. Cryoprotectants act by decreasing the freezing point of a substance, reducing the amount of salts and solutes present in the liquid phase of the sample and by decreasing ice formation within the spermatozoa.

Before cryopreservation semen samples are collected by masturbation after 2 or 3 days of abstinence and liquefied at room temperature. Usually, semen analysis is performed prior to processing for cryopreservation. There are two main freezing techniques used in sperm cryopreservation: slow freezing and rapid freezing (vitrification).

The slow freezing method may be manual or automated involving a semi-programmable freezer. It is performed by simultaneously decreasing the temperature of the semen while adding cryoprotectant in a stepwise manner and eventually plunging the samples into liquid nitrogen.

In particular, control of the cooling rate is often primitive Although sperm have been frozen successfully with a number of manual techniques, including the dropping of semen on to dry ice to form pellets and the holding of semen in the vapour phase of liquid nitrogen, significant differences in cooling rate between different samples can 
be a problem. The resulting straw-to-straw variation and loss of viability may not be important where sperm counts are normal, but in the case of oligozoospermic or asthenozoospermic samples these losses may be highly significant. Therefore, programmable freezers have proven valuable in reducing variability between freezes, despite the increased cost associated with the purchase of equipment.

Improvements in cryopreservation of human spermatozoa have been attempted in the past by the use of different cryoprotectants and extenders, and in particular, by altering the cooling rate, usually a linear reduction in temperature with time.

The cooling rate dependency of cell recovery of many cell types may be predicted from computer models of their osmotic behavior during freezing. However the predicted results with spermatozoa have not been in agreement with experimental observations. For example, although conventional models have suggested that human sperm cells should survive cooling rates up to $10000^{\circ} \mathrm{C} / \mathrm{min}$ (Noiles et al. 1993), experimentally the survival rate begins to decline beyond $100^{\circ} \mathrm{C} / \mathrm{min}$. It is clear that human spermatozoa have unusual cryobiological behavior and improvements in their survival have not been amenable to conventional approaches of cryobiology.

Many of the changes in physical properties which occur in an aqueous cryoprotectant following ice nucleation are not linear with temperature. Parameters such as the ice fraction, concentration of ionic species, osmolality, $\mathrm{pH}$, viscosity and gas solubility, all vary in a non-linear manner with temperature. In addition, the biophysical characteristics of cells which determine the response to freezing, for example the cellular permeability to water, also change in a non-linear manner with temperature.

In fact, in a previous study, Morris et al. (2002) proposed an ideal protocol for semen crypreservation. For this investigation, human spermatozoa suspended in a standard cryoprotectant were frozen using various protocols that manipulated different aspects of the physical conditions. The effects on post-thaw survival and function were assessed in comparison with conventional techniques. The results of this study demonstrated that the recovery of cryopreserved human spermatozoa can be significantly improved by controlling the concentration gradients experienced by the cells during freezing. When linear cooling was applied the recovery was significantly less than that achieved with controlled concentration and was similar to that reported in other studies.

The rapid freezing method, vitrification, is a process by which the samples are exposed to nitrogen vapour, equilibrated for some time and are then directly plunged into liquid nitrogen. It has been suggested that this rapid cooling would prevent the formation of sizeable intracellular ice crystals that are potentially lethal and the detrimental effects of high salt concentrations during freezing and rewarming (thawing). Similarly, the very fast warming rate would serve to prevent the recrystallization that may otherwise occur in the supercooled vitrified glass state. In addition, the entire vitrification process only takes a few seconds.

However, the conventional method of vitrification requires high cryoprotective agent's concentrations. It has been reported that the addition and removal of osmotically active cryoprotective agents during freezing and warming processes can induce lethal mechanical stress per se. Further problems include the chemical toxicity of cryoprotective agents and possible negative influence on the genetic apparatus of mammalian spermatozoa.

Therefore it has been suggested that it has not been possible to successfully cryopreserve the osmotically sensitive mammalian spermatozoon by conventional vitrification, which implies high cryoprotective agents concentrations and a relatively high $\left(\leq 5 \times 103^{\circ} \mathrm{C} / \mathrm{min}\right)$ cooling rate.

Nawroth et al.(2002) developed a technique of ice- and cryoprotective agents -free cryopreservation (vitrification) by direct plunging of a sperm suspension into liquid nitrogen. After storage, warming was achieved by direct melting of the frozen suspension. This freezing/warming method was performed at cooling and warming rates of up to hundreds of thousands of ${ }^{\circ} \mathrm{C} / \mathrm{min}$ and it was suggested that this simple, straightforward approach is able to preserve the ability of the spermatozoa to move and fertilize the oocyte. Its improved results over the conventional method of slow freezing may be attributed to avoiding the use of the classic permeable cryoprotectants, thus preventing the lethal effects of osmotic shock.

\section{SPERM CRYOINJURY}

Although the cryopreservation of sperm is an important routine technique used in the management of human male infertility, because of the damage associated with freezing, the motility of cryopreserved spermatozoa after thawing is statistically reduced with respect to prefreezing motility, and this factor also shows wide interindividual variability. The question of diminished spermatozoon motility after cryopreservation is crucial because this variable is known to be the first affected. However, the mechanism of sperm impairment and its mechanical and/or physical-chemical etiology remain unclear.

Mechanical cell injury by conventional freezing is a consequence of intracellular or extracellular ice crystal formation and osmotic damage due to extensive cell shrinkage. Subsequent rewarming and thawing of the cells can further deteriorate their viability through possible excessive osmotic swelling. As a result, average velocity in terms of the percentage of motile spermatozoa drops significantly after cryopreservation with respect to that of fresh sperm.

Cryoinjury is not limited to the freezing process but sperm quality may also be affected by the subsequent slow-thawing process. This process induces further cell damage. Ice crystals melt during thawing and could result in damage of the sperm organelles. Therefore, the thawing phase and cryoprotectant removal must be conducted in a stepwise manner, similar to freezing.

There are many risks associated with cryopreservation. Freezing to subzero temperatures causes irreversible injury in spermatozoa, which gets aggravated during the thawing procedure, thereby reducing the sperm cryosurvival rate. Cryoprotectants themselves can be toxic if used in high concentrations since spermatozoa are vulnerable to osmotic changes induced by cryoprotectants. Spermatozoa can also undergo excessive dehydration (due to increased solute concentration) and denaturation of proteins due to shifts in $\mathrm{pH}$ as well as membrane damage caused by close proximity freezing of cell.

The cooling rate plays an important role in determining the extent of cryoinjury to the spermatozoa. During freezing, ice nucleates in the extracellular matrix, eliciting an osmotic gradient. During freezing, water will move across the membrane from the intracellular to the extracellular space and intracellular ice formation will occur with rapid cooling rates. If the cooling rate is too slow, water can join the ice phase of the extracellular space and the cells become osmotically inactive due to intracellular ice formation and loss of cell membrane integrity.

Thus, the cooling rate is very critical. Fast temperature decrease will cause severe intracellular ice formation but too slow temperature decrease can expose the cells to toxicity damage by high solute concentrations. Sperm motility, plasma membrane integrity and mitochondrial function are inversely correlated with cooling rates, which indicates that too fast or too slow cooling rates can cause these parameters to be compromised (Van Blerkom and Henry 1992). Osmotic changes during the cryopreservation process may result in cellular damage. In relation to cryopreservation, it was demonstrated that survival of human spermatozoa was decreased at hypoosmotic conditions due to cellular swelling leading to lysis. Spermatozoa were initially resistant to hyperosmotic conditions; however, significant cell damage occurred when returned to isosmotic conditions (Meyers 2005). The membrane integrity is an important factor for the sperm motility and viability. Membrane fluidity has been positively correlated with the recovery of motile, viable spermatozoa from a cryopreserved sample. The integrity of the sperm membrane is affected during cryopreservation and thawing processes. The stability of the membrane is affected by 
changes in temperature, volume changes associated with the movement of water, cryoprotectants and osmotic stress due to increased salt concentration.

During cryopreservation, the initial cooling process causes extensive chemical and physical damage to sperm cell membranes due to changes in lipid-phase transition and/or increased lipid peroxidation. It is well established that the production of reactive oxygen species leads to an increase in lipid peroxidation after cryopreservation and that this event is associated with a loss of sperm motility. As previously suggested (Chatterjee and Gagnon 2001), the injury to human spermatozoa induced by conventional cryopreservation occurs mainly during thawing and has been related to diminished antioxidant defense activity during cooling and/ or structural damage to the cytoskeleton and/or antioxidant enzymes during cryopreservation.

Cryopreservation of human spermatozoa is also known to have negative effects on sperm motility and velocity due to acrosomal leakage and degeneration. It has been reported that irregular interaction between DNA and nuclear proteins can lead to impaired motion parameters in spermatozoa.

Normozoospermic semen samples appear to be more tolerant to damage induced by freezing and thawing compared with oligozoospermic samples. It was reported that motile spermatozoa could be recovered after five refreeze-thaw cycles in normozoospermic samples and after two refreezethaw cycles in oligozoospermic specimens samples (Verza Jr et al. 2009). It has also been reported that up to half of men presenting with testicular cancer or lymphoma have impaired semen quality (O'Connell et al. 2002) and some investigators have shown that damage to sperm cryopreserved from cancer patients is more severe than that from normal donors (Hallak et al. 2000).

However, other researchers have demonstrated a similar decline in quality after cryopreservation in control and cancer groups (Agarwal 2000). Rofeim and Gilbert (2005) demonstrated that sperm integrity remains unchanged after the initial cellular damage induced by the freezing process. Semen from men with lymphoma and testicular cancer also tolerated the cryopreservation well up to 5 years. In addition it was reported that refreezing of human semen by the technique of liquid nitrogen vapor allows the retrieval of viable spermatozoa after thawing in cancer patients (Verza and Esteves 2008).

Advances in cryopreservation techniques and better initial semen quality in cancer patients, perhaps due to early referral and semen collection before the start of gonadotoxic therapy, may explain an increased toleration of sperm to cryoinjury. Moreover, with advanced ARTs only few spermatozoa are needed to achieve fertilization. As Hallak et al. (1998) demonstrated, after a median storage time of 49 months for cancer patients, the pregnancy rate per cycle of ART was $36.4 \%$. This finding is significant for cancer patients where infertility can last up to 5 years after radiotherapy or chemotherapy.

\section{CRYOPRESERVATION PRIOR TO CANCER TREATMENT}

Cryopreservation is an integral component of fertility preservation management in cancer patients and much of its successful application will affect success rates of ARTs. As described above, freezing and thawing semen further reduces sperm count, motility and viability. Although, additional samples and longer abstinence periods may be used to achieve higher total sperm counts, the need to initiate lifesaving anti-cancer therapy urgently often becomes a barrier in the process.

Semen banking should ideally be done before the start of cancer treatment. Theoretically semen collection and storage is feasible after the initiation of chemotherapy and radiation therapy, at least until azoospermia ensues. However, it is advisable to wait for $12-18$ months because of the time taken for the recovery of spermatogenesis and significant increase in the frequency of sperm aneuploidy persisting for 18 months or more after initiation of anti-cancer treatment. Post-pubertal men are generally able to ejaculate, howe- ver, some young cancer patients may not be able to produce a sample by masturbation. A strong vibrator or a rectal electric probe can be used to stimulate ejaculation in these boys; however it should be used under anaesthesia to avoid pain. Another concern with electroejaculation is the possible reduction in sperm motility.

In 2006, the American Society of Clinical Oncology published guidelines recommending that oncologists discuss the possibility of infertility with reproductive-age cancer patients and offer referral for fertility preservation consultation and therapy (Lee et al. 2009).

Despite these measures, referral patterns are still inconsistent in many centers, even large multidisciplinary ones, and many reproductive-age patients still start treatment without discussion of or opportunity for fertility preservation. Nearly half $(45 \%)$ of oncologists surveyed at one large university medical about effective qualitative changes in this field center reported never referring patients to a reproductive endocrinologist for fertility consultation (Forman et al. 2009).

In fact, although it is well established that fertility in adult life may be severely impaired by gonadotoxic therapies, the awareness of most oncologists of this subject is still insufficient. Several reports have described the lack of knowledge on the part of oncologists about ART.

The success rates of IVF and ICSI treatments using cryopreserved semen are currently almost as high as those using fresh semen. However, it has been also reported that only a small proportion of cancer patients have sperm samples preserved before the beginning of cancer treatment. Several studies have suggested that factors such as lack of and/or inaccurate knowledge of infertility risk at the time of diagnosis contribute to the low frequency of sperm cryopreservation in the cancer group of patients. Moreover, some authors have raised doubts about the justification and necessity of providing the facilities for banking spermatozoa before chemotherapy (Radford et al. 1999) specially because of the relatively small number of men making use of it following completion of treatment is less relatively low. In a previous report on the use and outcome of cryopreserved semen of cancer patients, Van Casteren ey al. (2008) described an average success rate of achieving parenthood using cryopreserved semen of at least $54 \%$. Other studies reported a success rate ranging from 33\% to $73 \%$ (Revel et al. 2005).

In a recently published work (Bonetti et al. 2008), we retrospectively evaluated the semen characteristics and attitudes of male cancer patients who had sperm banked before cancer treatment. For this, 98 male cancer patients were referred to our center for sperm banking before receiving potential gonadotoxic therapy, chemotherapy, and/or radiotherapy. Patients were asked to collect semen samples a minimum of three times, except for those patients who started chemotherapy immediately after enrolment into the sperm cryopreservation program; those in the latter category collected only one or two samples.

Demographic parameters, semen characteristics, destination of sperm banked samples and questionnaires answered by the patients regarding cryopreservation time were evaluated. The cancer diagnoses were testicle $(56.1 \%)$, prostate $(15.3 \%)$, Hodgkin's lymphomas (9.2\%), non-Hodgkin's lymphomas $(7.1 \%)$, leukemia $(3.1 \%)$ and other malignancies $(9.2 \%)$. Patients with testicular cancer presented lower sperm concentration; however, there were no differences with the percentage of normozoospermic patients among cancer type groups. A shorter time between cancer diagnosis and sperm banking was observed for testicular and prostate cancer patients. Nevetheless, more than $80 \%$ of the sperm samples remain cryopreserved in our sperm bank.

In our study, testicular cancer patients more frequently requested sperm cryopreservation, followed by prostate cancer patients. Also, we found that the mean time from diagnosis of cancer to the semen collection to cryopreservation was 4.5 months, but this period for testicular and prostate cancer patients was shorter. Reasons for this may be a higher level of awareness of the need for sperm banking by the medical team treating patients with cancer 
of the reproductive organs, or by the patient himself, who then influences the awareness level of the cancer site regarding fertility issues.

However, despite of the fade of the cryopreserved samples, we recommend that all men who are about to receive cancer treatment that could impair fertility, should be advised that sperm cryopreservation is at this moment the only established and reliable method to preserve fertility. Moreover, semen can be stored for a reasonable long time without affecting pregnancy chance.

Cancer patients also need to be made aware of the financial costs involved in the semen cryopreservation and ART as the insurance companies will not always cover these costs.

\section{CONCLUSION}

Postcancer quality of life studies revealed male factor infertility as one of the most devastating long-term side effects of anticancer therapy. As cancer survival has increased largely during the last decades, oncologists should now be more aware of the long-term quality of life.

In fact, many young survivors of cancer want to have children in the future. They also often would consider adopting a child rather than living without children. However, some patients are not informed of the deleterious effects of cancer chemotherapy on spermatogenesis, and many patients do not know about the availability of sperm cryopreservation.

In recent years, new ART has substantially increased the chances of becoming a father using cryopreserved semen. Male cancer patients might have an impaired pretreatment semen quality, but this certainly should not rule out sperm banking, since with ICSI only a few motile spermatozoa are needed.

In conclusion, sperm cryopreservation, at this moment, is the only established and reliable method to preserve fertility in men. Cancer physicians should inform and strongly recommend sperm cryopreservation, to all men at risk of becoming infertile after receiving gonadotoxic.

\section{Correspondence}

Avenida Brigadeiro Luis Antônio, 4545

São Paulo - SP, Brasil

CEP: 01401-002

Rua Vieira Maciel, 62

CEP: 04503-040

edson@fertility.com.br

\section{REFERENCES}

Agarwal, A. (2000). "Semen banking in patients with cancer: 20-year experience." Int J Androl 23 Suppl 2: 16-9.

Alvarez, J. G. and Storey, B. T. (1993). "Evidence that membrane stress contributes more than lipid peroxidation to sublethal cryodamage in cryopreserved human sperm: glycerol and other polyols as sole cryoprotectant." J Androl 14(3): 199-209.

Blake, D. A., Farquhar, C. M., Johnson, N. and Proctor, M. (2007). "Cleavage stage versus blastocyst stage embryo transfer in assisted conception." Cochrane Database Syst $\operatorname{Rev}(4)$ : CD002118.

Boekelheide, K., Schoenfeld, H. A., Hall, S. J., Weng, C. C., Shetty, G., Leith, J., et al. (2005). "Gonadotropin-releasing hormone antagonist (Cetrorelix) therapy fails to protect nonhuman primates (Macaca arctoides) from radiation-induced spermatogenic failure." J Androl 26(2): 222-34.

Bonetti, T. C., Melamed, R. M., Braga, D. P., Madaschi, C., Iaconelli, A., Jr., Pasqualotto, F. F., et al. (2008). "Assisted reproduction professionals' awareness and attitudes towards their own IVF cycles." Hum Fertil (Camb) 11(4): 254-8.

Brennemann, W., Brensing, K. A., Leipner, N., Boldt, I. and Klingmuller, D. (1994). "Attempted protection of spermatogenesis from irradiation in patients with seminoma by D-Tryptophan-6 luteinizing hormone releasing hormone." Clin Investig 72(11): 838-42.

Brenner, H., Steliarova-Foucher, E. and Arndt, V. (2007). "Up-to-date monitoring of childhood cancer long-term survival in Europe: methodology and application to all forms of cancer combined." Ann Oncol 18(9): 1561-8.
Brook, P. F., Radford, J. A., Shalet, S. M., Joyce, A. D. and Gosden, R. G. (2001). "Isolation of germ cells from human testicular tissue for low temperature storage and autotransplantation." Fertil Steril 75(2): 269-74.

Bunge, R. G. and Sherman, J. K. (1953). "Fertilizing capacity of frozen human spermatozoa." Nature 172(4382): 767-8.

Carmely, A., Meirow, D., Peretz, A., Albeck, M., Bartoov, B. and Sredni, B. (2009). "Protective effect of the immunomodulator AS101 against cyclophosphamide-induced testicular damage in mice." Hum Reprod 24(6): 1322-9.

Chatterjee, S. and Gagnon, C. (2001). "Production of reactive oxygen species by spermatozoa undergoing cooling, freezing, and thawing." Mol Reprod Dev 59(4): 451-8.

Forman, E. J., Anders, C. K. and Behera, M. A. (2009). "Pilot survey of oncologists regarding treatment-related infertility and fertility preservation in female cancer patients." J Reprod Med 54(4): 203-7.

Hallak, J., Hendin, B. N., Thomas, A. J., Jr. and Agarwal, A. (1998). "Investigation of fertilizing capacity of cryopreserved spermatozoa from patients with cancer." J Urol 159(4): 1217-20.

Hallak, J., Mahran, A., Chae, J. and Agarwal, A. (2000). "Poor semen quality from patients with malignancies does not rule out sperm banking." Urol Res 28(4): 281-4.

Hovatta, O. (2001). "Cryopreservation of testicular tissue in young cancer patients." Hum Reprod Update 7(4): 378-83.

Lee, S. H., Song, H., Park, Y. S., Koong, M. K., Song, I. O. and Jun, J. H. (2009). "Poor sperm quality affects clinical outcomes of intracytoplasmic sperm injection in fresh and subsequent frozen-thawed cycles: potential paternal effects on pregnancy outcomes." Fertil Steril 91(3): 798-804.

Lirdi, L. C., Stumpp, T., Sasso-Cerri, E. and Miraglia, S. M. (2008). "Amifostine protective effect on cisplatin-treated rat testis." Anat Rec (Hoboken) 291(7): 797-808.

Masala, A., Faedda, R., Alagna, S., Satta, A., Chiarelli, G., Rovasio, P. P., et al. (1997). "Use of testosterone to prevent cyclophosphamide-induced azoospermia." Ann Intern Med 126(4): 292-5.

Meyers, S. A. (2005). "Spermatozoal response to osmotic stress." Anim Reprod Sci 89(1-4): 57-64.

Morris, I. D., Ilott, S., Dixon, L. and Brison, D. R. (2002). "The spectrum of DNA damage in human sperm assessed by single cell gel electrophoresis (Comet assay) and its relationship to fertilization and embryo development." Hum Reprod 17(4): 990-8.

Nawroth, F., Isachenko, V., Dessole, S., Rahimi, G., Farina, M., Vargiu, N., et al. (2002). "Vitrification of human spermatozoa without cryoprotectants." Cryo Letters 23(2): 93-102.

Noiles, E. E., Mazur, P., Watson, P. F., Kleinhans, F. W. and Critser, J. K. (1993). "Determination of water permeability coefficient for human spermatozoa and its activation energy." Biol Reprod 48(1): 99-109.

O'Connell, M., McClure, N. and Lewis, S. E. (2002). "The effects of cryopreservation on sperm morphology, motility and mitochondrial function." Hum Reprod 17(3): 704-9.

Okada, F. K., Stumpp, T. and Miraglia, S. M. (2009). "Carnitine reduces testicular damage in rats treated with etoposide in the prepubertal phase." Cell Tissue Res 337(2): 269-80.

Otala, M., Suomalainen, L., Pentikainen, M. O., Kovanen, P., Tenhunen, M., Erkkila, K., et al. (2004). "Protection from radiation-induced male germ cell loss by sphingosine-1-phosphate." Biol Reprod 70(3): 759-67.

Radford, J., Shalet, S. and Lieberman, B. (1999). "Fertility after treatment for cancer. Questions remain over ways of preserving ovarian and testicular tissue." Bmj 319(7215): 935-6.

Revel, A., Haimov-Kochman, R., Porat, A., Lewin, A., Simon, A., Laufer, N., et al. (2005). "In vitro fertilization-intracytoplasmic sperm injection success rates with cryopreserved sperm from patients with malignant disease." Fertil Steril 84(1): 118-22.

Rofeim, O. and Gilbert, B. R. (2005). "Long-term effects of cryopreservation on human spermatozoa." Fertil Steril 84(2): 536-7. 
Schover, L. R., Rybicki, L. A., Martin, B. A. and Bringelsen, K. A. (1999). "Having children after cancer. A pilot survey of survivors' attitudes and experiences." Cancer 86(4): 697-709.

Shetty, G. and Meistrich, M. L. (2005). "Hormonal approaches to preservation and restoration of male fertility after cancer treatment." J Natl Cancer Inst Monogr(34): 36-9.

Spitz, S. (1948). "The histological effects of nitrogen mustards on human tumors and tissues." Cancer 1(3): 383-98.

Van Blerkom, J. and Henry, G. (1992). "Oocyte dysmorphism and aneuploidy in meiotically mature human oocytes after ovarian stimulation." Hum Reprod 7(3): 379-90. van Casteren, N. J., van Santbrink, E. J., van Inzen, W., Romijn, J. C. and Dohle, G. R. (2008). "Use rate and assisted reproduction technologies outcome of cryopreserved semen from 629 cancer patients." Fertil Steril 90(6): 2245-50.

Verza Jr, S., Feijo, C. M. and Esteves, S. C. (2009). "Resistance of human spermatozoa to cryoinjury in repeated cycles of thaw-refreezing." Int Braz J Urol 35(5): 581-90; discussion 591.

Verza, S., Jr. and Esteves, S. C. (2008). "Sperm defect severity rather than sperm Source is associated with lower fertilization rates after intracytoplasmic sperm injection." Int Braz J Urol 34(1): 49-56. 